\title{
Efficient Downlink Channel Reconstruction for FDD Transmission Systems
}

\author{
Yu Han*, Tien-Hao Hsu ${ }^{\dagger}$, Chao-Kai Wen ${ }^{\dagger}$, Kai-Kit Wong ${ }^{\S}$, and Shi Jin* \\ *National Mobile Communications Research Laboratory, Southeast University, Nanjing, China \\ ${ }^{\dagger}$ Institute of Communications Engineering, National Sun Yat-sen University, Kaohsiung 804, Taiwan \\ ${ }^{\S}$ Department of Electronic and Electrical Engineering, University College London, United Kingdom
}

\begin{abstract}
In this paper, a novel scheme is proposed to reconstruct downlink channel for frequency-division-duplex (FDD) systems from uplink channel state information (CSI) with limited feedback. We adopt the Newtonized orthogonal matching pursuit (NOMP) algorithm to estimate the frequency-independent parameters of the delays of a multi-path channel through uplink training. The gain of each path is further refined through downlink training using least squares (LS) algorithm and sent back to the base station (BS). Using the uplink-estimated delays and the downlink-refined gains, the BS reconstructs the downlink multipath channel. Simulations and over-the-air tests are conducted to examine the performance of the proposed NOMP-LSbased reconstruction scheme for the downlink channel. Results confirm the effectiveness of the NOMP-LS scheme and that the reconstructed channel is accurate.
\end{abstract}

\section{INTRODUCTION}

Frequency-division-duplex (FDD) is one of the most popular duplexing modes in wireless communication systems where uplink and downlink transmissions co-exist simultaneously over two frequency bands. However, reciprocity does not exist between uplink and downlink channels, making the estimation of channel state information (CSI) non-trivial. Conventionally, downlink CSI is acquired through downlink training and then feedback in FDD systems. Due to the use of multi-antenna techniques, especially large-scale antenna arrays in the fifth generation $(5 \mathrm{G})$ mobile communication networks [1], this conventional downlink CSI acquisition method is no longer feasible. Therefore, new ideas and methods need to be sought.

In [2], [3], the authors utilized the slowly changing feature of the space and estimated the downlink CSI from the downlink training process. Numerical results in [4] also showed that a significant overhead reduction can be achieved via the partial support information obtained from the uplink. These results have given evidence to the possibility of exploiting the correlation between uplink and downlink for downlink CSI acquisition, which motivates the work of this paper.

In this study, we utilize the frequency-independent features of the delays of a multipath channel to reconstruct the downlink channel from uplink measurement. We do this by proposing a Newtonized orthogonal matching pursuit (NOMP)-least squares (LS)-based scheme. In our proposed approach, the base station (BS) first estimates the gains and the delays during the uplink training process using the NOMP algorithm [5], and then sends the estimated delays to the user. The user refines the gains through downlink training using LS estimation and afterwards sends these gains back to the BS. As a result, the downlink channel can be reconstructed based on the uplink-estimated delays and the downlink-refined gains. Computer simulations are conducted to assess the performance of the scheme and we find that we can reconstruct highly accurate in-band channel using NOMP. The refinement of the gains significantly improves the accuracy of the reconstructed out-of-band (OOB) channel. We conduct over-the-air (OTA) tests and reveal that the reconstructed channel resembles the minimum mean square error (MMSE)-estimated channel, even in non-line-of-sight (NLoS) indoor scenarios. The proposed scheme opens up a new direction for the downlink CSI acquisition solution of FDD massive MIMO systems.

\section{System MODEL}

A single cell of a mobile communication system is considered to function in the FDD transmission mode by employing orthogonal frequency-division multiplexing (OFDM). We focus on the baseband and regard the uplink carrier frequency as $0 \mathrm{~Hz}$. Then, the downlink carrier frequency is represented by its distance with the uplink carrier frequency, that is, $\triangle F$. Each of the uplink and downlink transmission bands is comprised of $N$ sub-carriers with spacing $\triangle f$, respectively. Both the BS and the user are equipped with a single antenna.

In the uplink, the time-domain received signal at the user side is expressed as

$$
Y_{\mathrm{u}}(t)=H_{\mathrm{u}}(t) * X_{\mathrm{u}}(t)+Z_{\mathrm{u}}(t),
$$

where $X_{\mathrm{u}}(t)$ is the transmit signal from the BS, $*$ denotes convolution, $Z_{\mathrm{u}}(t)$ is the additive noise, and $H_{\mathrm{u}}(t)$ is the timedomain expression of the multipath channel between the BS and the user. The uplink multipath channel is represented as

$$
H_{\mathrm{u}}(t)=\sum_{l=0}^{L-1} g_{\mathrm{u}, l} \delta\left(t-\tau_{u, l}\right),
$$

where $L$ is the number of propagation paths, $g_{\mathrm{u}, l}$ denotes the uplink complex gain of the $l$ th path, $\tau_{\mathrm{u}, l}=d_{u, l} / \mathrm{c}$ is the propagation time or time delay, $d_{\mathrm{u}, l}$ measures the propagation distance of the $l$ th path from the user to the BS, and $c$ is the speed of light. When transformed into the frequency domain, 
the uplink multipath channel is written as

$$
h_{\mathrm{u}}(f)=\sum_{l=0}^{L-1} g_{\mathrm{u}, l} e^{-j 2 \pi f \tau_{\mathrm{u}, l}},
$$

where $f$ denotes the frequency tone. Considering the baseband OFDM symbol before up conversion, the uplink channel on the $n$th sub-carrier reads

$$
h_{\mathrm{u}}(n)=\sum_{l=0}^{L-1} g_{\mathrm{u}, l} e^{-j 2 \pi n \triangle f \tau_{\mathrm{u}, l}},
$$

where $n=0, \ldots, N-1$.

Obviously, reciprocity does not exist between the uplink and downlink channels in FDD systems. However, the uplink and downlink channels share a common propagation space between the transmitter and receiver, and they further share the scatterers and propagation paths. In short, spatial reciprocity still exists. In [6], it was illustrated that the propagation distance of each propagation path is frequency-independent, indicating that $\tau_{\mathrm{u}, l}$ are common for the correlated frequency band and can be simplified to $\tau_{l}$. Thus, we can write the downlink multipath channel on the $n$th sub-carrier as

$$
h_{\mathrm{d}}(n)=\sum_{l=0}^{L-1} g_{\mathrm{d}, l} e^{-j 2 \pi(\triangle F+n \triangle f) \tau_{l}} .
$$

where $n=0, \ldots, N-1$.

\section{Downlink Channel ReConstruction}

As the BS needs to acquire downlink CSI to design proper transmission schemes such as user scheduling before downlink data transmission, here, we focus on the downlink CSI acquisition and propose a novel downlink channel reconstruction scheme, based on uplink CSI and limited feedback.

\section{A. Motivations}

The spatial reciprocity between the uplink and the downlink channels in FDD systems inspires us to reconstruct the downlink channel based on the frequency-independent parameters of the delays. Through the uplink training process, the BS is able to abstract these parameters from the received pilots. It was suggested in [6] that the gains are frequency-independent as well, and the downlink channel is possible to be inferred solely from the uplink estimated gains and delays. However, there is no OTA evidence to verify the frequency-independence of the gains. Completely eliminating the downlink training and feedback processes results in inaccurate downlink CSI, as shown in our numerical results in Section IV. Therefore, we suggest to refine the gains through downlink training and feedback. Since the NOMP algorithm is utilized in uplink training and the LS estimation is adopted in downlink refinement, the proposed scheme is referred to as the NOMP-LSbased downlink channel reconstruction scheme. The working process of the NOMP-LS-based reconstruction can be divided into three phases, which will be discussed in the following subsection.

\section{B. NOMP-LS-based Reconstruction}

1) Phase I - Extraction of frequency-independent parameters: We first calculate $\left\{g_{\mathrm{u}, l}, \tau_{l}\right\}_{l=0, \ldots, L-1}$ in the uplink because the BS can obtain the complete uplink CSI through uplink training process. The uplink pilots allocated for the user are uniformly inserted in every $\alpha$ sub-carriers, where $\alpha$ is a positive integer, and the pilot on each sub-carrier equals 1 . Then, the received pilots on all the allocated sub-carriers can be stacked to form a vector as

$$
\mathbf{y}_{\mathrm{u}}=\sum_{l=0}^{L-1} g_{\mathrm{u}, l} \mathbf{p}\left(\tau_{l}\right)+\mathbf{z}_{u},
$$

where $\mathbf{y}_{\mathrm{u}}$ and $\mathbf{z}_{\mathrm{u}}$ are $N / \alpha$-dimensional uplink received pilot and noise vectors, respectively; the $N / \alpha$-dimensional vector

$$
\mathbf{p}\left(\tau_{l}\right)=\frac{1}{\sqrt{N}}\left[e^{-j 2 \pi f_{0} \tau_{l}}, \ldots, e^{-j 2 \pi\left(f_{0}+(N-\alpha) \triangle f\right) \tau_{l}}\right]^{T}
$$

contains the delay of the propagation path, and $f_{0}$ is the frequency tone of the first sub-carrier allocated for the user.

In this study, the NOMP algorithm is adopted to estimate $g_{\mathrm{u}, l}$ and $\tau_{l}$. We first provide an brief overview to the NOMP algorithm proposed in [5]. NOMP is an iteration based algorithm which aims to minimize the cost function as follows

$$
S(g, \tau)=\left\|\mathbf{y}_{\mathrm{u}}-\sum_{i=0}^{l-1} g_{\mathrm{u}, l} \mathbf{p}\left(\tau_{l}\right)\right\|^{2} .
$$

During the $i$ th iteration, NOMP finds and updates the twotuples of $\left(g_{\mathrm{u}}, \tau\right)$ according to the following four steps.

- Identify: Coarsely estimate $\hat{\tau}_{i}$ from an over-sampled delay grid, and then calculate $\hat{g}_{\mathrm{u}, i}$ from $\hat{\tau}_{i}$.

- Single Refinement: Solely refine the coarse estimate twotuple $\left(\hat{g}_{\mathrm{u}, i}, \hat{\tau}_{i}\right)$ by the Newton update steps and add the obtained $\left(\hat{g}_{\mathrm{u}, i}^{\prime}, \hat{\tau}_{i}^{\prime}\right)$ into the set of the estimated two-tuples.

- Cyclic Refinement: Cyclically refine the set of the estimated two-tuples through the Newton update steps.

- Update: Retain the estimated delays and update all the gains through LS estimation.

The NOMP algorithm terminates when all the multipath components are estimated and only the noise is remained in the pilot signal model. We assume that there are $\hat{L}$ paths detected and denote the final results of the extracted frequencyindependent parameters as $\left\{\hat{g}_{\mathrm{u}, l}, \hat{\tau}_{l}\right\}_{l=0, \ldots, \hat{L}-1}$.

2) Phase II - Downlink gain refinement: The gains should be refined to achieve accurate downlink reconstructions, although we have obtained all the components required to reconstruct the downlink channel. Therefore, we further conduct the downlink training to re-estimate the gains of all the paths. A relatively minimal quantity of downlink pilots is required because of the insignificant estimation demand. Only one OFDM symbol is occupied by the downlink pilots. We use comb-type all-1 pilots and sparsely and uniformly insert them into the downlink transmission band. A pilot is inserted in every $\beta$ sub-carriers, where $\beta$ is a positive integer.

For the $k$ th occupied sub-carrier, where $k=0, \ldots, N / \beta-1$, 

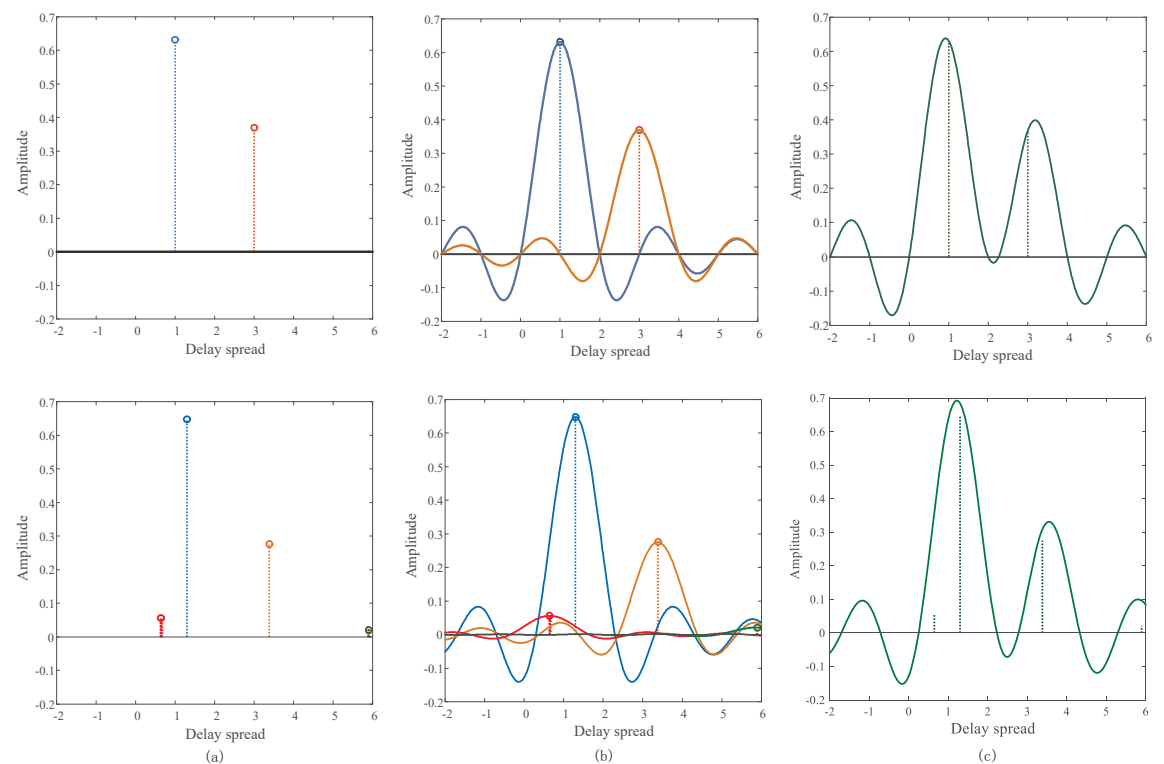

Fig. 1. Simulation results of the in-band reconstruction of the propagation paths. We first estimate the amplitudes and delays of the propagation paths as shown in (a). The reconstructed paths in frequency domain are then transformed into time domain (b) and composed to form (c).

the received pilot at the user side is expressed as

$$
y_{\mathrm{d}}(k)=\sum_{l=0}^{L-1} g_{\mathrm{d}, l} e^{-j 2 \pi f_{k} \tau_{l}}+z_{\mathrm{d}}(k),
$$

where $f_{k}=\triangle F+\beta k \triangle f$ is the frequency tone of the $k$ th occupied sub-carrier. Stacking all the downink received pilots into a vector, we obtain the following expression

$$
\mathbf{y}_{\mathrm{d}}=\mathbf{A g}+\mathbf{z}_{\mathrm{d}},
$$

where $\mathbf{y}_{\mathrm{d}}$ and $\mathbf{z}_{\mathrm{d}}$ are $N / \beta$-dimensional downlink received pilot and noise vectors, respectively; $\mathbf{g}=\left[g_{\mathrm{d}, 0}, \ldots, g_{\mathrm{d}, L-1}\right]^{T}$ is the stacked gain vector, $\mathbf{A}$ is the $N / \beta \times L$ dimensional coefficient matrix with the $(m, n)$ th entry satisfying

$$
\mathbf{A}_{m n}=e^{-j 2 \pi f_{m} \tau_{n}},
$$

where $m=0, \ldots, N / \beta-1, n=0, \ldots, L-1$. Since the BS knows $\left\{\hat{\tau}_{l}\right\}_{l=0, \ldots, \hat{L}-1}$ and informs the user about these estimates, A becomes a $\hat{L} N / \beta \times \hat{L}$ matrix with all the elements known to the user by applying the estimates. Then, we can obtain the LS estimation of the gains by using

$$
\hat{\hat{\mathrm{g}}}=\mathbf{A}^{\dagger} \mathbf{y}_{\mathrm{d}}
$$

where $\mathbf{A}^{\dagger}$ represents the pseudo-inverse of $\mathbf{A}$ and $\hat{\mathrm{g}}$ remains an $\hat{L}$-dimensional vector.

3) Phase III - Downlink channel reconstruction: The downlink-refined gains are sent back to the BS. Utilizing the uplink-estimated delays and the downlink-refined gains, the BS reconstructs the downlink channel as

$$
\hat{h}_{\mathrm{d}}(f)=\sum_{l=0}^{\hat{L}-1} \hat{\hat{g}}_{\mathrm{d}, l} e^{-j 2 \pi f \hat{\tau}_{l}},
$$

where $f$ denotes the frequency tone to be reconstructed.

\section{Performance Evaluation}

In this section, we evaluate the performance of the NOMPLS-based downlink channel reconstruction scheme. The uplink and downlink reconstructions are examined through in-band and $\mathrm{OOB}$ reconstructions, respectively. We follow the Verizon 5G standard [7] and set the full bandwidth as $153.6 \mathrm{MHz}$, with 2048 sub-carriers and $75 \mathrm{kHz}$ sub-carrier space. The results for computer simulations and OTA tests are presented.

\section{A. Simulation Results}

When conducting the simulations, we regard the central 45 $\mathrm{MHz}$ around the direct current (DC) as the in-band and the rest of the frequency band as the OOB.

We first examine the NOMP-based in-band reconstruction. Fig. 1 compares the norm of the gains, that is, the amplitudes of the practical and the reconstructed channels, where the top three sub-figures correspond to the practical paths, and the bottom three sub-figures refer to the reconstructed paths. Two original paths exist in the practical propagation scenario. By applying the NOMP algorithm to the in-band pilots, we could derive four paths. The second and third paths highly approximate the original paths in delay and amplitude, while the first and fourth derived paths are inexistent in practice. Although the number of the derived paths are different from the practical channel, this error causes a negligible difference to the reconstruction result. The reconstructed in-band channel is close to the practical channel from the superpositions of the paths shown in the two right-side sub-figures. The NOMP algorithm behaves well in in-band channel reconstruction.

Then, we evaluate the effect of the LS-based refinement on the OOB reconstruction. Fig. 2(a) illustrates the difference between the practical full-band channel and the inferred channel which is solely derived from the in-band NOMP results. 


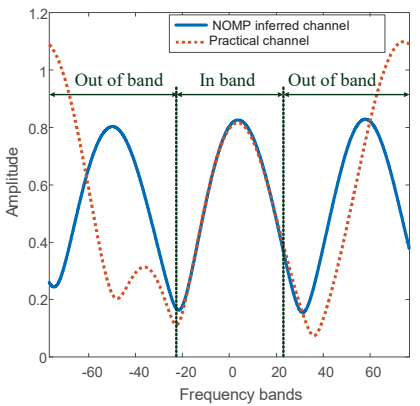

(a)

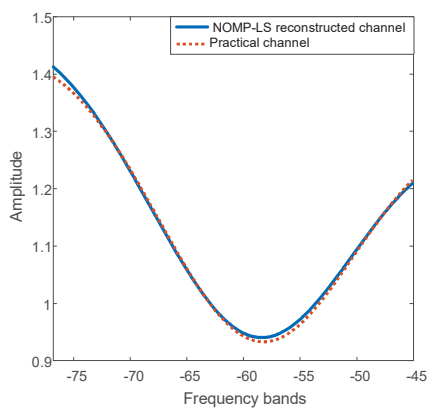

(b)

Fig. 2. Simulation results of the full-band reconstruction, where (a) presents the inferred channel that is resulted from the in-band estimates, and (b) shows the reconstructed channel of the NOMP-LS-based scheme.

The $45 \mathrm{MHz}$ in-band reconstructed channel coincides with the true channel. However, we can observe an obvious deviation for the OOB between the inferred and the practical channels. The large gap reveals that the gains derived from in-band estimation are insufficiently accurate in inferring the OOB channel. Therefore, we refine the gains through OOB training and LS estimation. We set $\beta=4$, that is, pilots are inserted in every four sub-carriers. The results of the refinement are depicted in Fig. 2(b). The reconstructed OOB channel is near the practical channel, thereby validating the necessity and effectiveness of the refinement. The NOMP-LS-based OOB reconstruction achieves equal accuracy with the NOMP-based in-band construction. If we increase the density of the OOB pilots, then the accuracy will be further improved.

\section{B. OTA Test Results}

The NOMP-LS-based reconstruction scheme is also evaluated via OTA tests. The hardware system is established mainly by the SGT100A radio frequency vector signal generator and the RTO2000 digital oscilloscopes. The OTA test environment is depicted in Fig. 3, where the yellow circle represents the position of the transmitter, and the red squares are the possible positions of the receiver. Positions (1) and (2) are selected to test the LoS and NLoS scenarios, respectively.

Fig. 4 illustrates the in-band and OOB of the OTA tests. The central $45 \mathrm{MHz}$ bandwidth around DC is still regarded as the in-band. The $31.8 \mathrm{MHz}$ bandwidth in blue or orange is the $\mathrm{OOB}$, which central frequency is $60.9 \mathrm{MHz}$ away from the DC. In the gain refinement phase, we set $\beta=4$, and 106 sub-carriers are allocated for the OOB pilots. The MMSE-

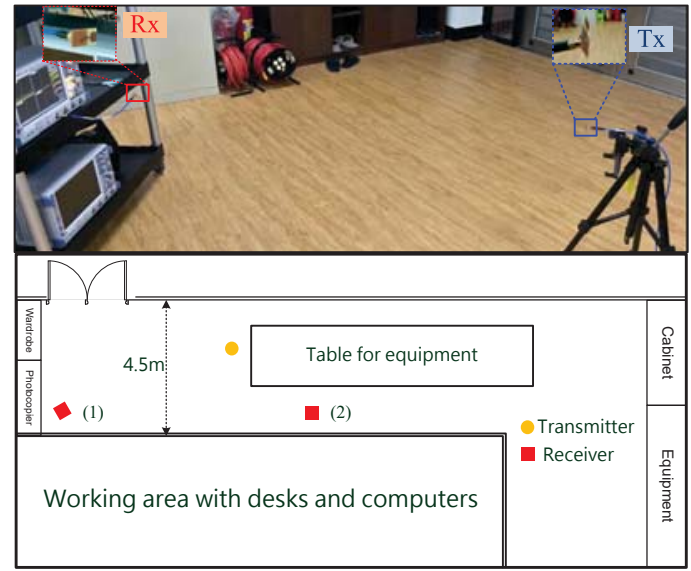

Fig. 3. OTA test environment. Positions (1) and (2) correspond to the LoS and NLoS scenarios, respectively.

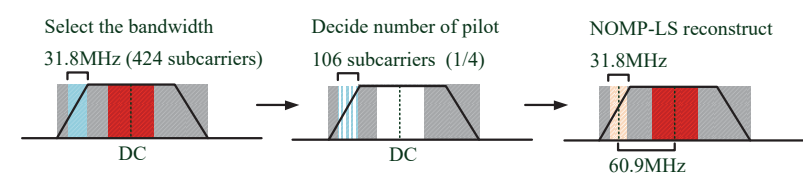

Fig. 4. Illustration of the in-band (red or white regions) and OOB (blue or orange regions) in the OTA tests.

estimated channel is regarded as the real channel to evaluate the performance of the NOMP-LS-based scheme. We use the mean square error (MSE) as the metric, which is expressed as

$$
\mathrm{MSE}=\frac{\mathbb{E}\left\{\left\|\hat{h}_{\mathrm{MMSE}}-\hat{h}_{\mathrm{NOMP}-\mathrm{LS}}\right\|^{2}\right\}}{\sigma_{n}^{2}},
$$

where $\hat{h}_{\mathrm{MMSE}}$ and $\hat{h}_{\mathrm{NOMP}-\mathrm{LS}}$ are the MMSE-estimated channel and NOMP-LS reconstructed channel, respectively, and $\sigma_{n}^{2}$ is the variance of the noise. The test results are displayed in the form of cumulative distribution function (CDF).

We first test the case when the receiver is located at Position (1). Fig. 5(a) provides the CDF of the MSE (in dB). We can observe that the NOMP-based in-band reconstruction achieves the highest accuracy, with a $90 \%$ probability that the MSE is below $-9.65 \mathrm{~dB}$. However, the performance of the NOMP-based OOB reconstruction is poor, with $90 \%$ probability MSE equaling $15.36 \mathrm{~dB}$. The results demonstrate that in-band channel can be directly derived from the in-band training process while the performance of the NOMP-based $\mathrm{OOB}$ reconstruction is poor. If we infer the OOB channel solely from the in-band CSI, the reconstructed channel will not accurately determine the practical channel. Fortunately, the accuracy is greatly improved when the gains are refined through the OOB training process. The NOMP-LS-based OOB reconstruction functions well, with a $90 \%$ probability that the MSE is below $-6.64 \mathrm{~dB}$. The OTA results align well with the simulation results. We can further observe the power ratio of the propagation paths through Fig. 5(b). The first reconstructed path occupies $75.2 \%$ power of the channel because the LoS 


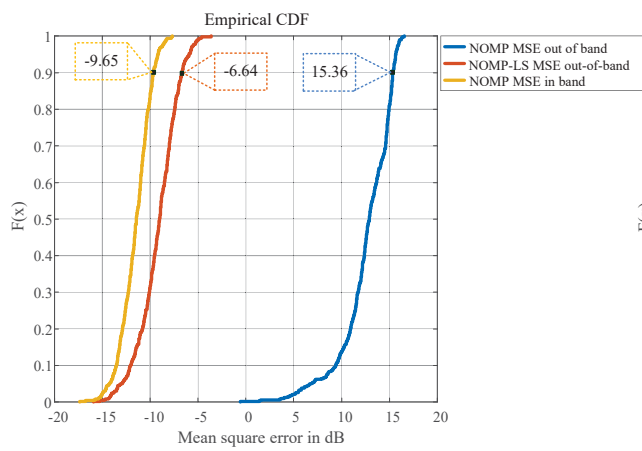

(a)

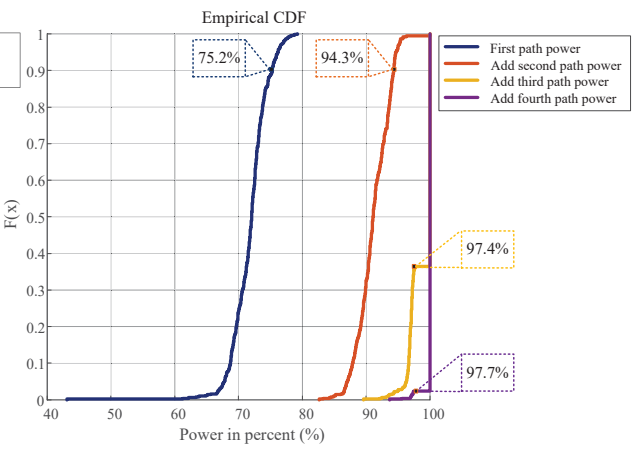

(b)

Fig. 5. MSE of the NOMP-LS-based channel reconstruction and the power ratio of reconstructed paths when conducting OTA tests in Position (1).

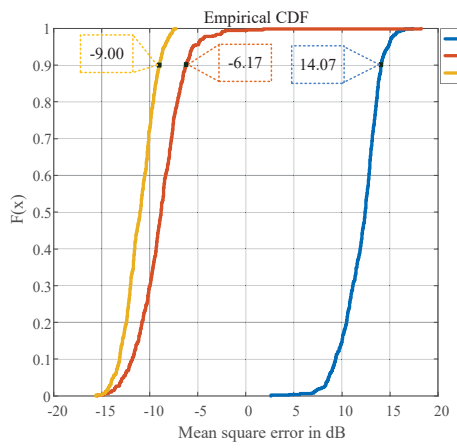

(a)

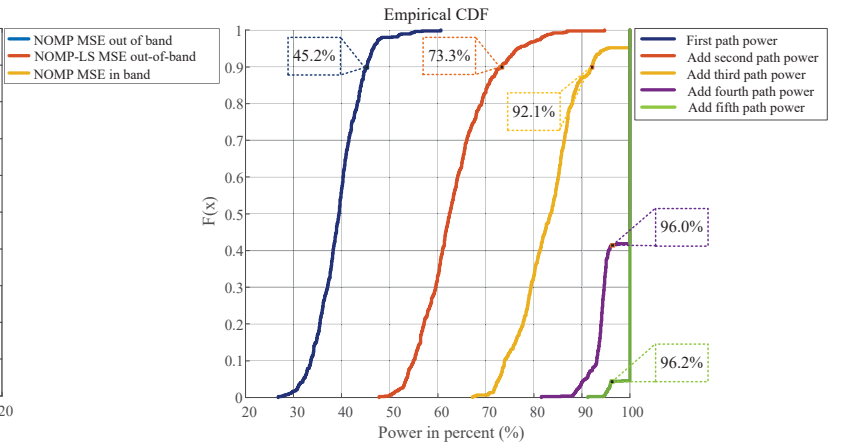

(b)

Fig. 6. MSE of the NOMP-LS-based channel reconstruction and the power ratio of reconstructed paths when conducting OTA tests in Position (2).

propagation path exists between the transmission and the Position (1). The power ratio increases to $94.3 \%$ after adding the second reconstructed paths. The channel is mainly composed of the two propagation paths.

Fig. 6 illustrates the OOB reconstruction results of Position (2). We find that the first two reconstructed paths only occupy $73.3 \%$ power of the channel. The NOMP-based in-band and the NOMP-LS-based OOB reconstructions still have excellent performance in the NLoS propagation scenario. The 90\%probability MSEs of the two reconstructions are $-9.00 \mathrm{~dB}$ and $-6.17 \mathrm{~dB}$, which are not considerably inferior to the results of the LoS case. These results strongly demonstrate that the proposed scheme functions well even when multiple NLoS paths exist in the channel.

\section{CONCLUSion}

In this paper, an NOMP-LS scheme based on the frequencyindependent features of the delays of the paths was proposed to reconstruct the downlink channel for FDD transmission systems. These delays were first calculated using the NOMP algorithm during uplink training. Then the gains were refined via downlink training and feedback to achieve accurate downlink reconstructions. The simulation and OTA test results have validated its effectiveness for in-band and OOB channel reconstructions. The proposed scheme has also been proven to function well even in NLoS propagation scenarios.

\section{ACKNOWLEDGEMENT}

The work of S. Jin was supported by the National Science Foundation (NSFC) for Distinguished Young Scholars of China with Grant 61625106 and the NSFC with Grant 61531011. The work of C.-K. Wen was supported in part by the Ministry of Science and Technology of Taiwan under Grant MOST 1062221-E-110-019, and in part by ITRI, Hsinchu, Taiwan.

\section{REFERENCES}

[1] E. Larsson, O. Edfors, F. Tufvesson, and T. L. Marzetta, "Massive MIMO for next generation wireless systems," IEEE Commun. Mag., vol. 52, no. 2, pp. 186-195, 2014.

[2] J. Choi, D. J. Love, and P. Bidigare, "Downlink training techniques for FDD massive MIMO systems: Open-loop and closed-loop training with memory," IEEE J. Sel. Topics Signal Process., vol. 8, no. 5, pp. 802-814, 2014.

[3] W. Shen, L. Dai, B. Shim, Z. Wang, and R. W. Heath, "Channel feedback based on AoD-adaptive subspace codebook in FDD massive MIMO systems," arXiv preprint arXiv:1704.00658, 2017.

[4] J. Shen, J. Zhang, E. Alsusa, and K. B. Letaief, "Compressed CSI acquisition in FDD massive MIMO: How much training is needed?" IEEE Trans. Wireless Commun., vol. 15, no. 6, pp. 4145-4156, 2016.

[5] B. Mamandipoor, D. Ramasamy, and U. Madhow, "Newtonized orthogonal matching pursuit: Frequency estimation over the continuum," IEEE Trans. Signal Process., vol. 64, no. 19, pp. 5066-5081, 2016.

[6] D. Vasisht, S. Kumar, H. Rahul, and D. Katabi, "Eliminating channel feedback in next-generation cellular networks," in Proc. ACM SIGCOMM, Aug. 2016, pp. 398-411.

[7] Cisco, Ericsson, Intel Corp., LG Electronics, Nokia, Qualcomm Technologies Inc., Samsung Electronics, and Verizon, "Physical channels and modulation (Release 1)," http://www.5gtf.net/V5G_211 v1p $7 \cdot \mathrm{pdf}$. 